\title{
Colony-stimulating factor 1 receptor inhibition blocks macrophage infiltration and endometrial cancer cell proliferation
}

\author{
FU HUA ${ }^{1,2}$, YE TIAN ${ }^{1,3}$, YINGCHUN GAO ${ }^{2},{\text { CHANGHUA } \text { LI }^{2} \text { and XIAOPING LIU }}^{2}$ \\ ${ }^{1}$ Department of Radiotherapy and Oncology, The Second Affiliated Hospital of Soochow University, Soochow University, \\ Suzhou, Jiangsu 215004; ${ }^{2}$ Department of Gynecology, Huai'an First People's Hospital, Nanjing Medical University, Huai'an, \\ Jiangsu 223300; ${ }^{3}$ Department of Basic Research, Institute of Radiotherapy and Oncology, Soochow University, \\ Suzhou, Jiangsu 215004, P.R. China
}

Received November 28, 2017; Accepted January 25, 2019

DOI: $10.3892 / \mathrm{mmr} .2019 .9963$

\begin{abstract}
Tumor-associated macrophages (TAMs) promote the progression of endometrial cancer (EC), but the mechanism of TAM in EC cell proliferation remains unclear. It was found that colony stimulating factor (CSF)-1 and CSF-1 receptor (CSF-1R) were highly expressed in EC tissues of patients and two EC cell lines (ECC-1 and HEC-1A). Using wound-healing and chemotactic migration assays to evaluate the role of EC cells in the induction of macrophage migration, it was found that the supernatant of EC cells promoted macrophage cell line (U937) migration; however, the migration capacity of U937 weakened when CSF-1R was blocked. Subsequently, inhibition of CSF-1 expression in EC cells also restrained U937 migration. Additionally, blocking CSF-1R by PLX3397 treatment in U937 cells inhibited EC cell proliferation in a co-culture system by inhibiting the expression of proliferation-associated proteins (Janus kinase-1, phosphoinositide 3-kinase, AKT, cyclin kinase 2, 4 and retinoblastoma-associated protein). Together, these results demonstrated that CSF-1 secreted by EC cells promoted macrophage migration; similarly, CSF-1-stimulated macrophages promoted EC cell proliferation. These results suggested that the interaction between CSF-1 and its receptor served an important role in promoting macrophage infiltration and progression of EC.
\end{abstract}

\section{Introduction}

Endometrial cancer (EC) is one of the three most common malignancies in the female reproductive system. With the application of estrogen, increased obesity and dietary changes in recent years, the incidence of EC has increased (1). For

Correspondence to: Dr Ye Tian, Department of Radiotherapy and Oncology, The Second Affiliated Hospital of Soochow University, Soochow University, 1055 Sanxiang Road, Suzhou, Jiangsu 215004, P.R. China

E-mail: dryetian@126.com

Key words: tumor-associated macrophages, endometrial cancer, colony-stimulating factor 1 , colony-stimulating factor 1 receptor patients with metastatic tumor, there is no effective treatment until now, Until now, there is no new treatment available. Standard treatment consists of primary hysterectomy and bilateral salpingo-oophorectomy, often using minimally invasive approaches (laparoscopic or robotic). Lymph node surgical strategy is contingent on histological factors (subtype, tumor grade, involvement of lymphovascular space), disease stage (including myometrial invasion), patients' characteristics (age and comorbidities), and national and international guidelines. Adjuvant treatment is tailored according to histology and stage. Various classifications are used to assess the risks of recurrence and to determine optimum postoperative management (2). Therefore, an in-depth study of the role and mechanism of EC metastasis is very important for the effective treatment of EC. Tumor-associated macrophages (TAMs) are a group of cells with immunosuppressive functions, and are important components of the tumor microenvironment. TAMs regulate tumorigenesis, development and metastasis in a variety of ways $(3,4)$. The proliferation of TAMs and the secretion of inflammatory factors have promoted the development of type I EC (5). The density of TAMs in type II endometrial carcinoma is almost twice that of type I EC, and this difference may be due to the predominant result of M1 macrophages in the type II EC (6). The chemokine (C-X-C motif) ligand 8 secreted by TAM downregulates estrogen receptor alpha expression in EC cells by acting on homeobox B 13 protein may is involved in cancer invasion (7). TAMs also is involved in the production of neovascularization of EC and the infiltration of the myometrium by cancer cells (8). In a study of macrophage reactivity in 98 patients with primary EC, $\sim 40 \%$ of colony stimulating factor-1 (CSF-1)-expressing EC cells underwent infiltration of a large number of macrophages, suggesting that CSF-1 expression promotes the progression of endometrial cancer, and CSF-1 levels are closely associated with the degree of malignancy of primary tumors and their corresponding lymph node metastases (9). Therefore, CSF-1 may be involved in macrophage infiltration and the development of EC.

CSF-1 binds to the CSF-1 receptor (CSF-1R), which is expressed on the surface of macrophages, induces proliferation and infiltration of TAMs, and is involved in tumor cell proliferation, invasion and migration (10). A previous study found 
that CSF-1 is highly expressed in multiple types of tumors, and CSF-1 levels in the blood circulation could be used as a molecular marker for lung cancer, breast cancer, prostate cancer and lymphoma (11). A previous study reported that CSF-1R inhibitors can significantly reduce the volume of glioblastoma and tumor invasion by inhibiting TAMs, which indicates that there is an inflammatory cytokine interaction between TAMs and glioblastoma, and the role of mutual protection is presence between EC cells and TAMs (12). Thus, overexpression of CSF-1 or CSF-1R is positively associated with aggressive and poor prognosis of EC, and blocking CSF-1 or CSF-1R may inhibit EC progression. However, the mechanism of CSF-1 and CSF-1R pathway that regulates tumor invasion, tumor immunity and tumor angiogenesis is unclear. In the present study, through inhibiting the expression of CSF-1 and blocking CSF-1R, it was demonstrated that CSF-1 or CSF-1R inhibition may serve a similar role in blocking macrophage migration and EC cell proliferation.

\section{Materials and methods}

Patients. The records of 10 patients with endometrial carcinoma and 10 patients with endometrial benign lesions who underwent primary surgery at Huai'an First People's Hospital, Nanjing Medical University (Huai'an, China) between January 2016 and December 2016 were reviewed retrospectively. Inclusion criteria: Age, 35-65 years old; no hormone drug treatment for the past three months. Patients with non-endometrioid carcinoma (clear cell carcinoma and serous adenocarcinoma) and those who had received preoperative chemotherapy were excluded. The present study was approved by the Medical Ethics Committee of the Nanjing Medical University and all patients provided their written consent.

Reverse transcription-quantitative polymerase chain reaction $(R T-q P C R)$. Total RNA was extracted from endometrial tissue or EC cell lines (Cell bank, Shanghai Institute for Biological Science, China) using TRIzol ${ }^{\circledR}$ reagent (Life Technologies; Thermo Fisher Scientific, Inc., Waltham, MA, USA). After the RNA pellet was resuspended in diethyl pyrocarbonate-treated $\mathrm{H}_{2} \mathrm{O}$, the purity and concentration were determined by spectrophotometry, and the final concentration was adjusted to $1 \mu \mathrm{g} / \mu \mathrm{l}$. Reverse transcription was performed using a PrimeScript $^{\mathrm{TM}}$ II 1st Strand cDNA Synthesis kit (Takara Bio, Inc., Otsu, Japan) according to the manufacturer's instructions (5XRT Master Mix, $1 \mu \mathrm{g}$ RNA and Nuclease-free Water). The thermocycling conditions were $37^{\circ} \mathrm{C}, 15 \mathrm{~min} ; 50^{\circ} \mathrm{C}, 5 \mathrm{~min}$ and $98^{\circ} \mathrm{C}, 5$ min (then held at $4^{\circ} \mathrm{C}$ ), and cDNA was used as a template for PCR amplification of the target mRNA fragments. In order to detect the relative expression levels of CSF-1, CSF-1R, Janus kinase 1 (JAK-1), phosphoinositide 3-kinase (PI3K), AKT, cyclin-dependent kinase (CDK) 2, CDK4 and retinoblastoma-associated protein $(\mathrm{Rb}), \mathrm{SYBR}^{\circledR}$ Premix $E x$ $\mathrm{Taq}^{\mathrm{TM}}$ (Tli RNaseH Plus) kit (Takara Biotechnology, Co., Ltd., Dalian, China) was used for the RT-qPCR reaction, and the thermocycling conditions were $60 \mathrm{sec}$ at $95^{\circ} \mathrm{C}$, followed by 40 cycles of $15 \mathrm{sec}$ at $95^{\circ} \mathrm{C}, 15 \mathrm{sec}$ at $60^{\circ} \mathrm{C}$ and $45 \mathrm{sec}$ at $72^{\circ} \mathrm{C}$. A comparative quantification cycle $(\Delta \mathrm{Cq})$ method was used to quantify target mRNAs (13) and GAPDH was used as internal reference gene. PCR primers were: CSF-1, forward
5'-TGTGGTTTGTGGGAAAGCAG-3', reverse 5'-CTTCAG GCTCCTCTCTCTGG-3'; CSF-1R, forward 5'-GAGGAT GCTGTCCTGAAGGT-3', reverse 5'-GTACAGGCTCCC AGAAGGTT-3'; JAK-1, forward 5'-ATGGCCAGATGACAG TCACA-3', reverse 5'-TGTCCGATTGGATGGTTGGA-3'; PI3K, forward 5'-AGAAGCCTTCCTCTGTGTCC-3', reverse 5'-TCTTGCACAGCATCTCGTTG-3'; AKT, forward 5'-CTT TCGGCAAGGTGATCCTG-3', reverse 5'-GTACTTCAG GGCTGTGAGGA-3'; CDK2, forward 5'-GCCTTATGAGGC AGGTGAGA-3', reverse 5'-GTAGGAGGTGGACGTCAG AG-3'; CDK4, forward 5'-ACCGTTTACAAGGCCAGAGA-3', reverse 5'-ATCATGGGCCTCAGGTGAAA-3'; Rb, forward 5'-TTCCAGACCCAGAAGCCATT-3', reverse 5'-TCTGGG TGCTCAGACAGAAG-3'; and GAPDH, forward 5'-CTTTGT CAAGCTCATTTCCTGG-3', reverse 5'-TCTTGCTCAGTG TCCTTGC-3'.

ELISA. Endometrial tissue $\left(1-2 \mathrm{~cm}^{3}\right)$ was collected from 10 patients with endometrial benign lesions and from 10 patients with EC. Supernatants from the 20 cases of endometrial tissues (PBS and protease inhibitor were added to the tissue, and tissue homogenizer and sonicator were used to obtain supernatants) and cultured T-HESC, ECC-1, HEC-1A cells (density $>90 \%$ ) which were all cultured in Dulbecco's modified Eagle's medium (DMEM; Invitrogen; Thermo Fisher Scientific, Inc.) supplemented with $10 \%$ heat-inactivated fetal bovine serum (FBS; Sigma-Aldrich; Merck KGaA) at $37^{\circ} \mathrm{C}$ in a $95 \%$ humidified atmosphere of $95 \%$ air and $5 \% \mathrm{CO}_{2}$ were collected to measure the concentrations of CSF-1 by microplate reader, according to the manufacturer's protocol (DY216; R\&D systems, Inc., Minneapolis, MN, USA). Briefly, after normal endometrial tissue and EC tissue were homogenized and cultured cell supernatants were collected, the total protein concentration was determined by BCA Protein Assay kit (P0010S; Beyotime Institute of Biotechnology, Shanghai, China). According to the manufacturer's protocol, the protein supernatant was diluted at different dilutions (1:1,1:5 and 1:10), and the concentration of CSF-1 was calculated in different tissues and cells on the basis of the concentration of protein standards.

Western blot analysis. Western blot analysis was performed as previously described (14). Briefly, total protein (5 mg) was extracted from endometrial tissue or cultured cells $\left(2 \times 10^{6}\right)$ using radioimmunoprecipitation lysis buffer (Sangon Biotech, Co., Ltd., Shanghai, China) and total protein concentration was determined by BCA Protein Assay kit (P0010S; Beyotime Institute of Biotechnology). Primary polyclonal anti-rabbit CSF-1R (67455, 1:2,000), anti-rabbit JAK-1 (29261, 1:2,000), anti-rabbit PI3K (4249, 1:1,500), anti-phosphorylated rabbit (p)-AKT (4060, 1:2,000), anti-rabbit CDK2 (2546, 1:1,000), anti-rabbit CDK4 (12790, 1:1,000) and anti-rabbit p-Rb (8516, 1:1,000) were all purchased from Cell Signaling Technology Inc. (Danvers, MA, USA), and anti-GAPDH antibodies (AF0006, 1:5,000) were purchased from Beyotime Institute of Biotechnology. The primary antibody was added to the membrane for incubation overnight at $4^{\circ} \mathrm{C}$. The membrane was washed three times for $5 \mathrm{~min}$ with TBST at room temperature. Following a secondary horseradish peroxidase (HRP)-conjugated goat anti-rabbit (A0208) or goat anti-mouse 
(A0216) immunoglobulin (Ig)G antibody (1:5,000, Beyotime Institute of Biotechnology) incubation for $2 \mathrm{~h}$ at room temperature, signals were detected by the EasyBlot Chemiluminescence kit (Sangon Biotech, Co., Ltd.) and quantified using Image-Pro Plus 6.0 software (Media Cybernetics, Inc, Rockville, MD, USA).

Cell culture and immunocytochemistry staining. U937, T-HESC, HEC-1A and ECC-1 cell lines (Cell bank, Shanghai Institute for Biological Science, China) were all cultured in Dulbecco's modified Eagle's medium (DMEM; Invitrogen; Thermo Fisher Scientific, Inc., Waltham, MA, USA) supplemented with $10 \%$ heat-inactivated fetal bovine serum (FBS; Sigma-Aldrich; Merck $\mathrm{KGaA}$ ) at $37^{\circ} \mathrm{C}$ in a $95 \%$ humidified atmosphere of $95 \%$ air and $5 \% \mathrm{CO}_{2}$. In U937 and $\mathrm{EC}$ co-culture system, $100 \mathrm{U} / \mathrm{ml} \mathrm{M}-\mathrm{CSF}$ (Beyotime Institute of Biotechnology) were added to the culture medium at $37^{\circ} \mathrm{C}$ for $24 \mathrm{~h}$ to induce M2 type macrophages. Once U937 cells were successfully induced to M2 macrophages (expressing Arg-1 and CD206), they were used in subsequent experiments. Macrophage subtype was confirmed by inducible nitric oxide synthase (iNOS; 610328; BD Biosciences, Franklin Lakes, NJ, USA) and CD86 (6553689; BD Biosciences; double positive of M1 macrophage) and Arginase (Arg-1; PA5-29645; Thermo Fisher Scientific, Inc.) and CD206 (MA5-16868; Thermo Fisher Scientific, Inc.; double positive of M2 macrophage). Prior to CSF-1R immunofluorescence staining or crystal violet staining, cells were fixed with $4 \%$ paraformaldehyde for $15 \mathrm{~min}$ at room temperature. Primary mouse CSF-1R monoclonal antibody (sc46662, Santa Cruz Biotechnology, Inc., Dallas, TX, USA; 1:100) were revealed with specific goat anti-mouse Alexa Fluor ${ }^{\circledR} 555$ (IgG H\&L)-conjugated secondary antibodies (A0473; 1:50; Beyotime Institute of Biotechnology) for $1 \mathrm{~h}$ at $37^{\circ} \mathrm{C}$. Ki67 antibody (AF1738; 1:100; Beyotime Institute of Biotechnology) was used to examine the proliferation of EC cells. According to the conventional fluorescent staining process, goat anti-mouse Alexa Fluor ${ }^{\circledR} 555$ secary antibodies were used to examine the numbers of Ki67-positive EC cells. Subsequently, the cell morphology of U937 or EC cells were observed under a fluorescence microscope. Crystal violet purchased from Beyotime Institute of Biotechnology and was used to stain U937 cells for $15 \mathrm{~min}$ at room temperature.

ECC-1 or HEC-1A cell transfection. CSF-1-specific small interfering (si)RNA (siCSF-1; sense, 5'-ACGUGGCUAAAGUGU UAAAG-3'; antisense, 5'-CCUGUUCUGCAGUUCCUUCCU UGU-3'), and its corresponding non-silencing negative control siRNA (siNeg; sense, 5'-GGCAAAUUGCCCUUAUCCA-3'; antisense, 5'-AACGUUUAAACCGGUUACGUA3') were designed and synthesized by GeneChem, Inc. (Shanghai, China). ECC-1 or HEC-1A cells (50\% seeding density) were cultured in DMEM supplemented with $10 \%$ heat-inactivated fetal calf serum (Thermo Fisher Scientific, Inc.) at $37^{\circ} \mathrm{C}$ under $5 \% \mathrm{CO}_{2}$ in a humidified incubator. Cells in the exponential growth phase were grown for $24 \mathrm{~h}$ and then transfected using Lipofectamine ${ }^{\circledR} 3000$ (Thermo Fisher Scientific, Inc.), according to the manufacturer's protocol. The concentration of siCSF-1 and siNeg were maintained at $100 \mathrm{nM}$. The culture medium was replaced with DMEM plus $10 \% \mathrm{FBS}$ after $6 \mathrm{~h}$ of transfection, then $10 \mu \mathrm{g} / \mathrm{ml}$ of puromycin was added at $37^{\circ} \mathrm{C}$ for $24 \mathrm{~h}$ for screening positive cells, and $2 \mathrm{ug} / \mathrm{ml}$ puromycin were added to the cell culture medium to maintain positive cell selection. ECC-1 or HEC-1A cells transfected with siCSF-1 and siNeg were used for wound healing assay and Chemotactic migration assay.

CCK- 8 assay. Cell proliferation or growth rate was evaluated with the CCK-8 kit (Beyotime Institute of Biotechnology). U937 cells (1x104/well) were cultured in 96-well plates, or U937 cells $\left(5 \times 10^{4} /\right.$ well) were co-cultured with T-HESC $\left(5 \times 10^{4} /\right.$ well), ECC-1 $\left(5 \times 10^{4} /\right.$ well $)$ or HEC-1A $\left(5 \times 10^{4} /\right.$ well $)$ which were seeded in 24-well Transwell plates (Corning Inc., Corning, NY, USA), with U937 cells cultured on the upper chamber. After adding different concentrations of CSF-1 (10, 50, 100, 250 and $500 \mathrm{U} / \mathrm{ml}$ ) and $10 \mu \mathrm{M}$ PLX3397 into the culture medium on the upper chamber, cells were incubated with $20 \mu \mathrm{l} \mathrm{CCK}-8$ for $1 \mathrm{~h}$ at $37^{\circ} \mathrm{C}$, according to the manufacturer's protocol. Cell proliferation or growth rate was analyzed using a microplate reader to determine the absorbance at $450 \mathrm{~nm}$ (Biotec, Dresden, Germany).

Wound-healing assay. U937 cell migratory ability was measured using the wound-healing assay. Briefly, U937 cells were seeded in 6-well plates and grown to $90 \%$ confluency. The medium was replaced with cell (T-HESC, ECC-1 or HEC-1A) culture supernatant or fresh medium containing $100 \mathrm{U} / \mathrm{ml}$ CSF-1 or $10 \mu \mathrm{M}$ PLX3397; PBS was used as a vehicle control. Following the fresh medium exchange, a scratch was created with a $20 \mu \mathrm{l}$ pipette tip and an image was captured immediately. After $24 \mathrm{~h}$ incubation at $37^{\circ} \mathrm{C}$, images were captured again and the cell migration was quantified randomly by counting cells that had moved above the reference line. The control group was calculated as 100 and the experimental group compared with the control group to ascertain the difference between the groups.

Chemotactic migration assay. Chemotactic migration of U937 cells was measured using a Transwell chamber with $6.5 \mathrm{~mm}$ polycarbonate membrane $(8 \mu \mathrm{m}$ pore size) (Corning Inc.). Normal U937 cells $\left(5 \times 10^{4} /\right.$ well) with fresh medium containing $10 \mu \mathrm{M}$ PLX3397 or $100 \mathrm{U} / \mathrm{ml} \mathrm{CSF-1} \mathrm{were} \mathrm{plated}$ on the upper chamber, ECC-1 or HEC-1A cells $\left(5 \times 10^{4} /\right.$ well) that were stably transfected with CSF-1 interference plasmid (siCSF-1) or control plasmid (siNeg), were cultured on the lower chamber. The U937 cells were loaded at $1 \times 10^{5}$ cells/well to the upper chambers and allowed to migrate for $16 \mathrm{~h}$ at $37^{\circ} \mathrm{C}$. The cells were subsequently fixed with $4 \%$ paraformaldehyde at room temperature for $15 \mathrm{~min}$ and stained with crystal violet at room temperature for $10 \mathrm{~min}$, non-migrating cells on the upper surface of the membrane were removed using a cotton swab. The cells that had migrated to the lower surface of the membrane were randomly counted $\left(5 \times 10^{3} /\right.$ well) under a brightfield microscope.

Statistical analysis. Statistical analysis was performed using SPSS 18.0 software (SPSS Inc., Chicago, IL, USA). Data are presented as the mean \pm standard deviation of three independent experiments. One-way analysis of variance was used to determine statistical significance of differences between groups followed by Turkey post hoc test for comparison of two 
A

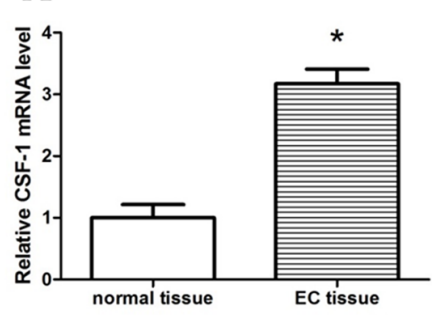

D

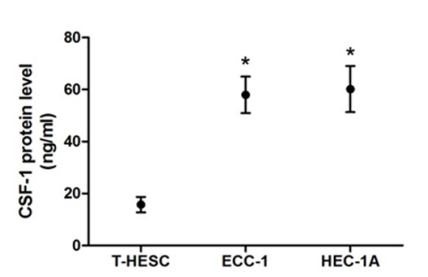

E

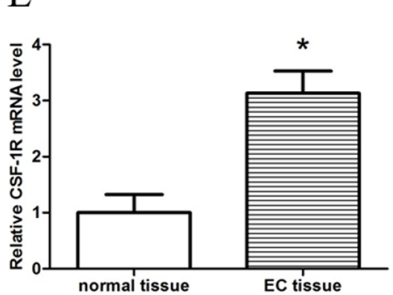

G

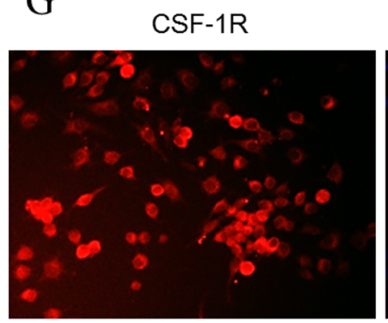

F

B

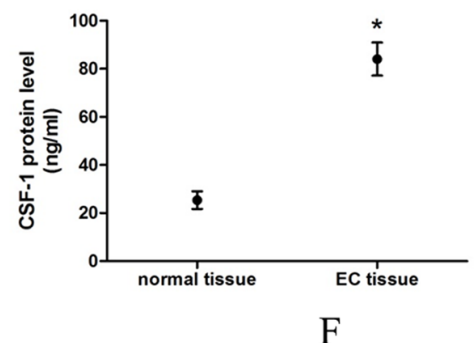

C

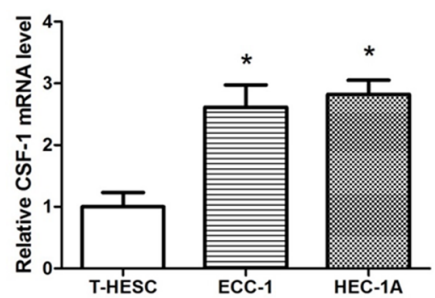

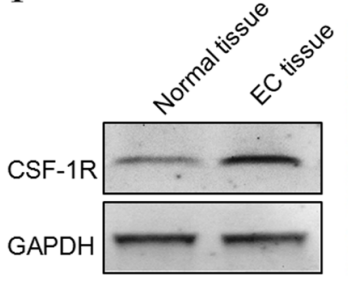

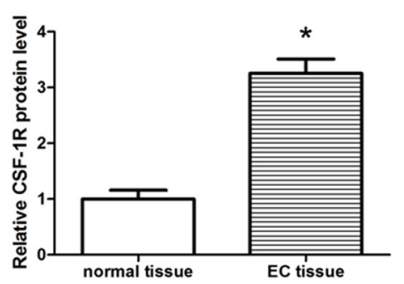

${ }^{H}$

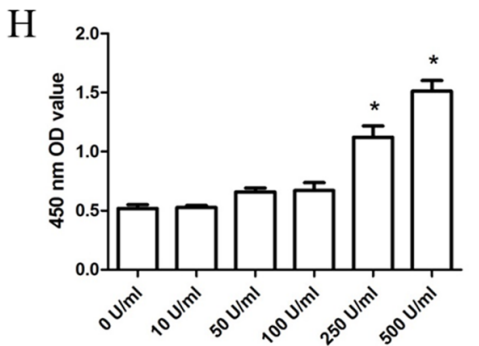

Figure 1. Expression levels of CSF-1 and CSF-1R in EC cells and macrophages. (A) mRNA and (B) protein expression levels of CSF-1 in endometrial tissues. (C) mRNA and (D) protein expression levels of CSF-1 in normal endometrial cells (T-HESC) and EC cells (ECC-1 and HEC-1A). (E) mRNA and (F) protein expression levels of CSF-1R in endometrial tissues. (G) Immunofluorescence detected CSF-1R expression levels in U937 cell lines. (H) Cell counting kit-8 assay detected the proliferation of U937 cells stimulated by different concentrations of CSF-1. Data are presented as the mean \pm standard deviation from 10 (tissues) or 5 (cells) independent experiments; "P<0.05 vs. Control. Scale bar, $100 \mu \mathrm{m}$. CSF, colony-stimulating factor; CSF-1R, colony-stimulating factor 1 receptor; EC, endometrial cancer; OD, optical density.

or more groups. $\mathrm{P}<0.05$ was considered to indicate a statistically significant difference.

\section{Results}

Expression of CSF-1 and CSF-1R in EC cells and macrophages in vivo and in vitro. In order to investigate CSF-1 expression in endometrial tissue and corresponding cells, RT-qPCR and ELISA were used to detect the RNA and protein expression levels, respectively, of CSF-1 in normal endometrial tissue, EC tissue and T-HESC, ECC-1 and HEC-1A EC cells. It was found that not only RNA but also protein expression levels in EC tissues and cell lines were significantly higher compared with the respective expression levels in normal endometrial tissue and T-HESC cells (Fig. 1A-D). Similar CSF-1, the protein expression levels of CSF-1R were significantly higher in EC tissue compared with normal tissue (Fig. 1E and F). In order to further investigate the expression of CSF-1R in macrophages, immunofluorescence staining was used to observe the expression of CSF-1R in U937 cell lines (Fig. 1G), which demonstrated that CSF-1R was highly positive on U937 cell membrane. CSF-1 and CSF-1R were both expressed in normal endometrial tissue and corresponding cells, especially in EC tissue. These results suggested that EC cells may recruit macrophages by secreting CSF-1. In order to examine the function of CSF-1, different concentrations of CSF-1 (10, 50, 100 and $500 \mathrm{U} / \mathrm{ml}$ ) were used to treat $\mathrm{U} 937$ cell, and proliferation was investigated. The result demonstrated that CSF-1 concentrations $>250 \mathrm{U} / \mathrm{ml}$ promoted the proliferation of U937 cells (Fig. 1H). Therefore $100 \mathrm{U} / \mathrm{ml} \mathrm{CSF-1} \mathrm{which} \mathrm{did} \mathrm{not} \mathrm{promote}$ cell proliferation was used in order to further observe the induction of macrophage migration by EC cells.

EC cells promote macrophage migration by secreting $C S F-1$. In order to observe whether EC cells could promote macrophage migration, EC cell supernatant was used for wound-healing and migration assay. In a wound-healing assay, the U937 culture medium was replaced with the supernatant from T-HESC, ECC-1 or HEC-1A cell cultures following a scratch on U937 cells. ECC-1 and HEC-1A supernatant treatment promoted more U937 cells to migrate compare with normal endometrial cell line T-HESC (Fig. 2A), which indicated that the supernatant of ECC-1 and HEC-1A cells may contain some components that are capable of promoting the migration of U937 cells. The CSF-1R inhibitor PLX3397 was added to the culture supernatant of U937 cells to observe whether CSF-1R is a key protein affecting U937 cell migration. It was found that the number of migrated U937 cells decreased when CSF-1R was blocked (Fig. 2A), which suggested that CSF-1 in ECC-1 and HEC-1A culture supernatant may be the key 
A
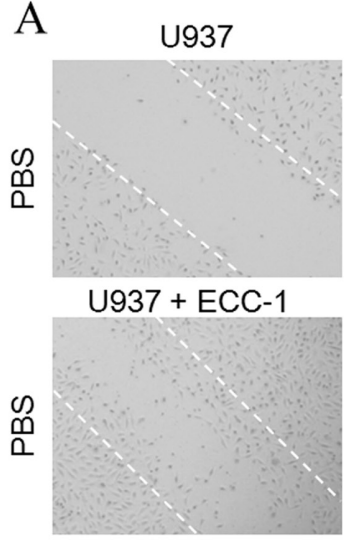

U937 + ECC-1
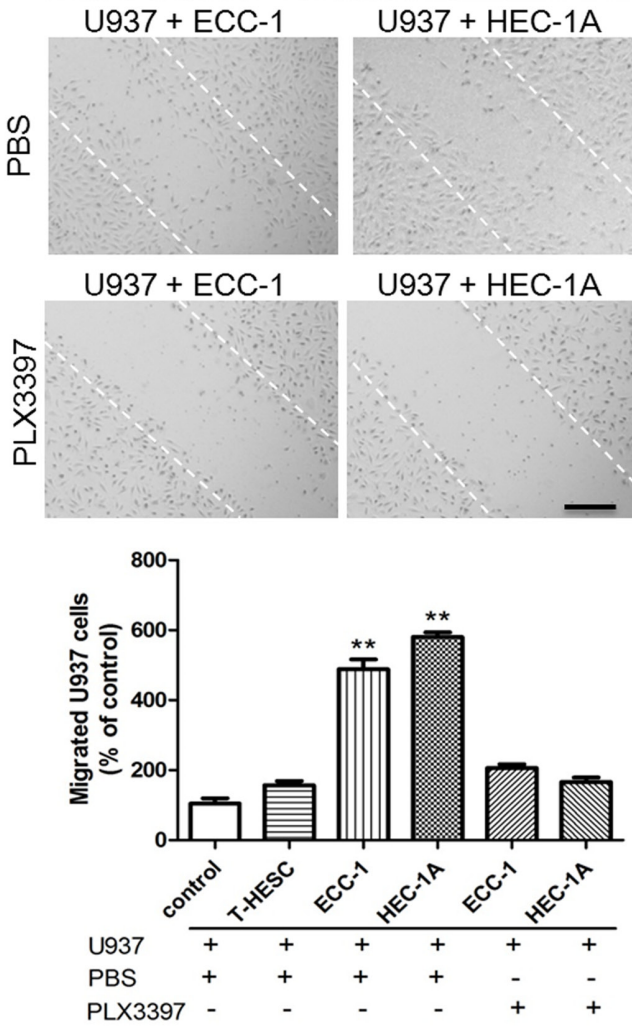

U937 + HEC-1A
B
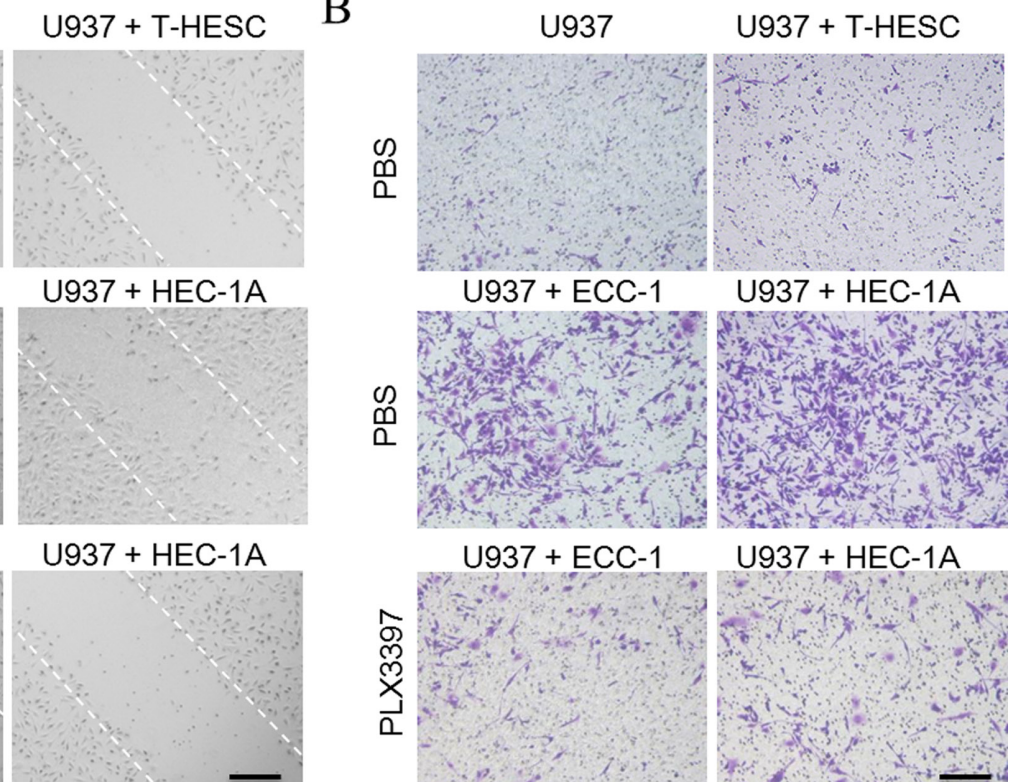

U937 + HEC-1A

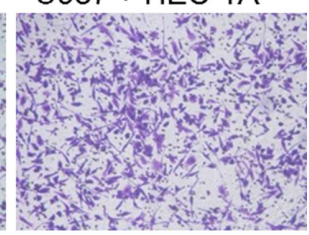

U937 + ECC-1
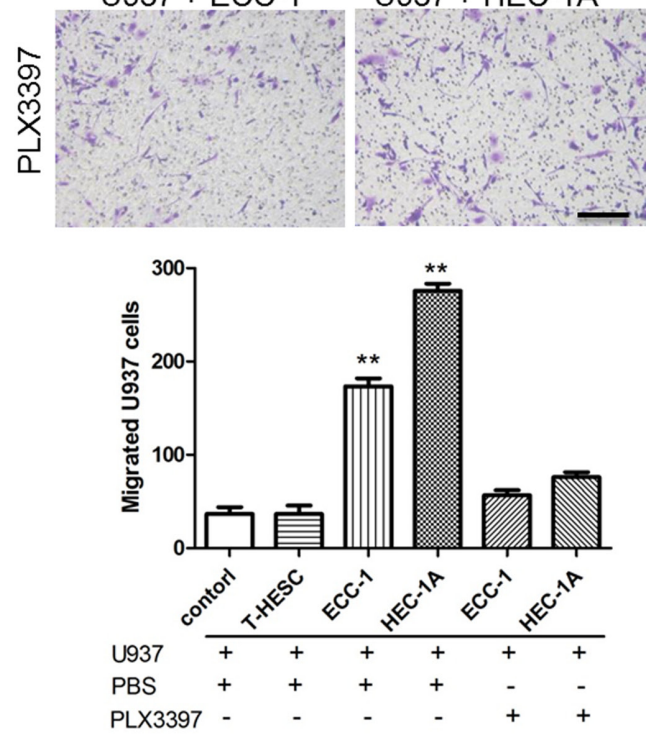

Figure 2. CSF-1R inhibition blocks U937 cell migration. (A) Wound-healing assay detected U937 cell migration. (B) Chemotactic migration assay detected U937 cells migration. Data are presented as the mean \pm standard deviation from 5 independent experiments; ${ }^{* *} \mathrm{P}<0.01 \mathrm{vs}$. control. Scale bar, $25 \mu \mathrm{m}$. CSF-1R, colony-stimulating factor 1 receptor.

molecule that induces U937 cell migration. Additionally, a chemotactic migration assay was used to confirm the role of CSF-1 in promoting U937 cell migration, and it was demonstrated that EC cells induced U937 cell migration, which was significantly reduced by blocking the CSF-1R on macrophages (Fig. 2B).

To further confirm the role of CSF-1 in inducing macrophage migration, $100 \mathrm{U} / \mathrm{ml} \mathrm{CSF-1} \mathrm{was} \mathrm{directly} \mathrm{added} \mathrm{to}$ U937 cell culture supernatant, and it was shown that CSF-1 may promote U937 cell migration, as measured by a wound healing (Fig. 3A) and a chemotactic migration assay (Fig. 3B). In order to further confirm the role of CSF-1, the expression of CSF-1 in ECC-1 and HEC-1A cells was reduced by siCSF-1 transfection; RT-qPCR and ELISA assay were used to examine the transfection efficiency of the siCSF-1 plasmid and confirmed the expression of CSF-1 was significantly decreased in transfected ECC-1 and HEC-1A cells (Fig. 3C). When the expression of CSF-1 in EC cells was silenced, the supernatant of ECC-1 and HEC-1A cells also failed to promote U937 cell migration (Fig. 3D and E). These results indicated that CSF-1 secreted by EC cells may be a key factor that promotes macrophage migration.
EC cells induce U937 cells into M2 macrophages (TAMs), which promote the proliferation of EC cells. In order to investigate the effects of macrophages migrating to EC tissue and whether macrophages affected the proliferation of EC cells, macrophages and EC cells were co-cultured. In the co-cultured system, EC cells were first cultured in 24-well plates and U937 cells cultured on coverslips respectively. When cell density reached the experimental requirements, the coverslip was moved into the 24-well to establish a co-culture system. U937 and ECC-1 cell lines were co-cultured in vitro for $24 \mathrm{~h}$, and makers of M1 macrophage [inducible nitric oxide synthase (iNOS) and CD86] and M2 macrophage [Arginase (Arg-1) and CD206] in U937 cell lines were investigated. iNOS and CD86 expressions in U937 cell lines were low, whereas Arg-1 and CD206 showed high expression in U937 cell lines (Fig. 4A). These data indicated that U937 were induced into M2 macrophages at $24 \mathrm{~h}$ culture. Subsequently, whether TAM had a role of promoting EC cell proliferation in this co-culture system was investigated, and it was found that the proliferation rate of EC cells (ECC-1 and HEC-1A) was increased, whereas U937 cells did not promote normal endometrial cell (T-HESC) proliferation (Fig. 4B). When PLX3397 was added to U937 
A
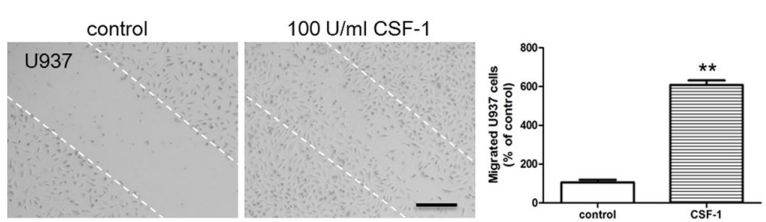

B

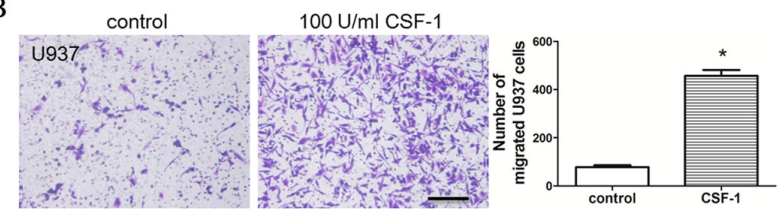

$\mathrm{C}$
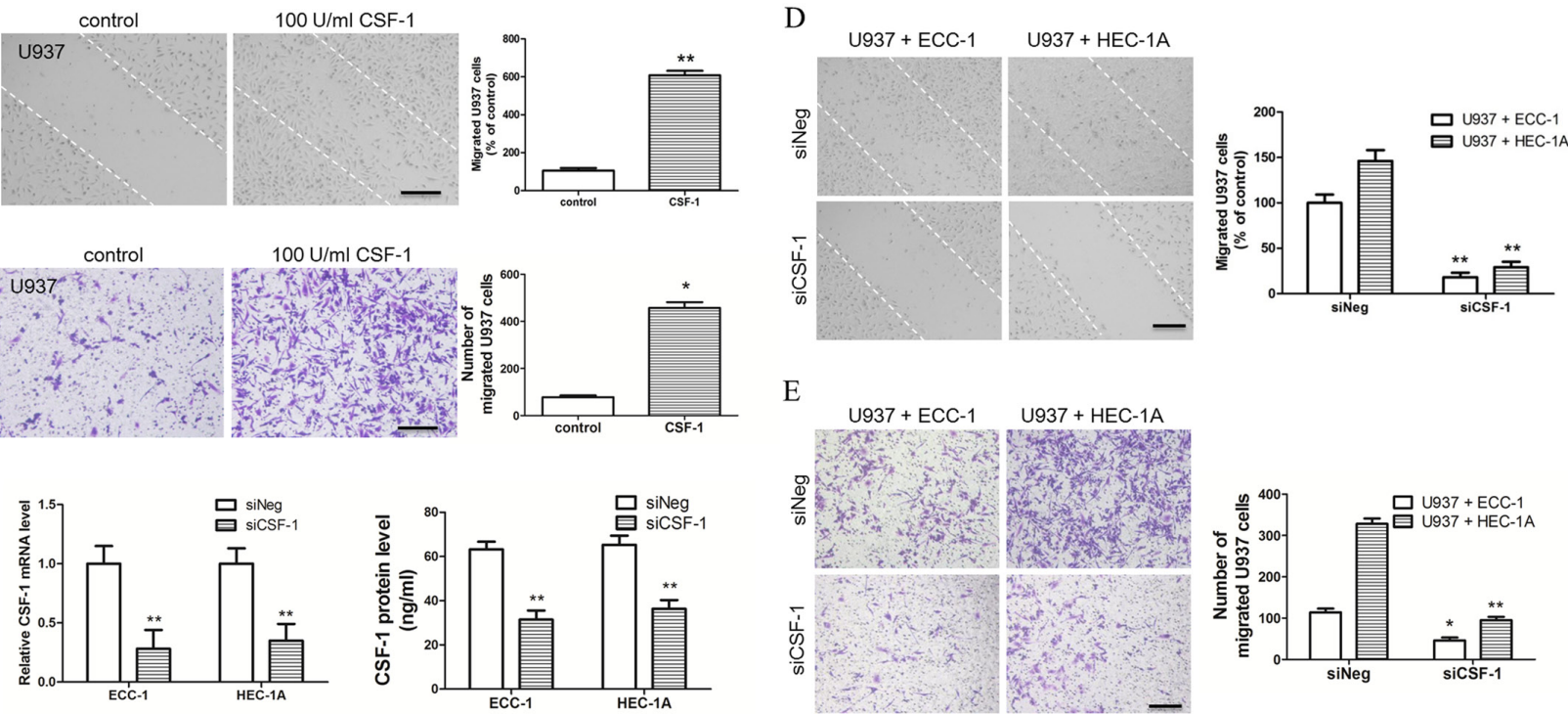

Figure 3. CSF-1 promotes U937 cell migration. (A) Wound-healing assay and (B) chemotactic migration assay found that CSF-1 promotes U937 cells migration following treatment with $100 \mathrm{U} / \mathrm{ml}$ CSF-1. (C) U937 cells were efficiently transfected with si-CSF-1. (D) Wound healing assay and (E) chemotactic migration assay confirmed that CSF-1 promotes U937 cells migration in ECC-1 and HEC-1A cells transfected with si-CSF-1. Data represent the mean \pm standard deviation from 5 independent experiments; ${ }^{*} \mathrm{P}<0.05,{ }^{* *} \mathrm{P}<0.01$ vs. Control. Scale bar: $25 \mu \mathrm{m}$. CSF-1, colony-stimulating factor 1; Neg, negative control; si, small interfering.
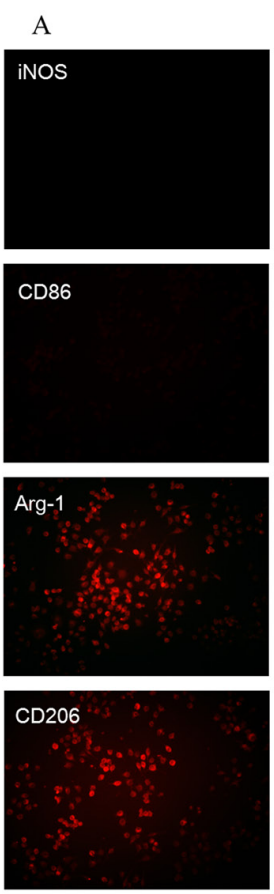
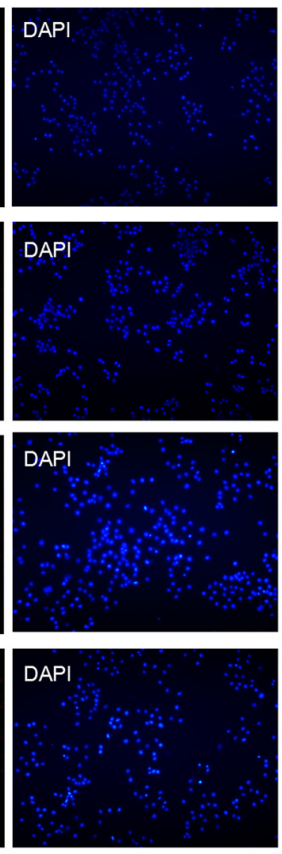
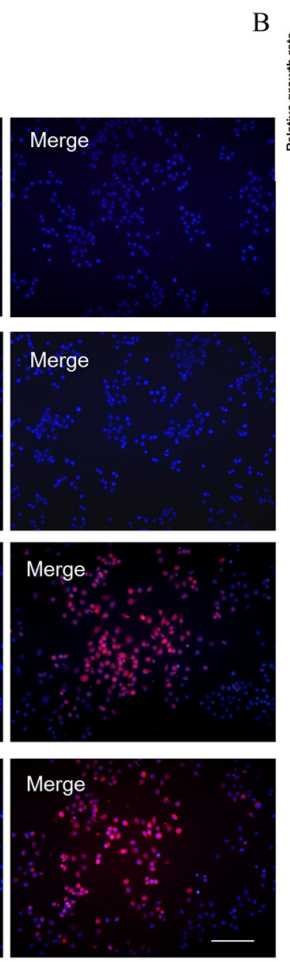
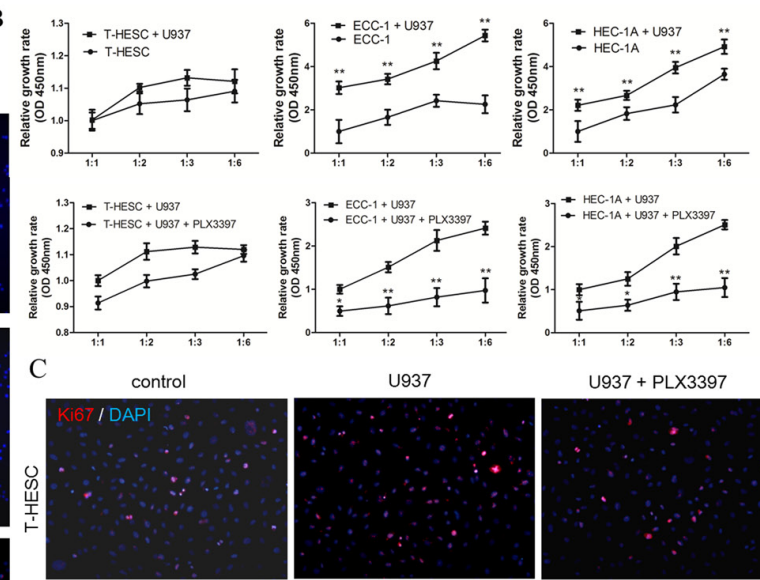

U937 + PLX3397
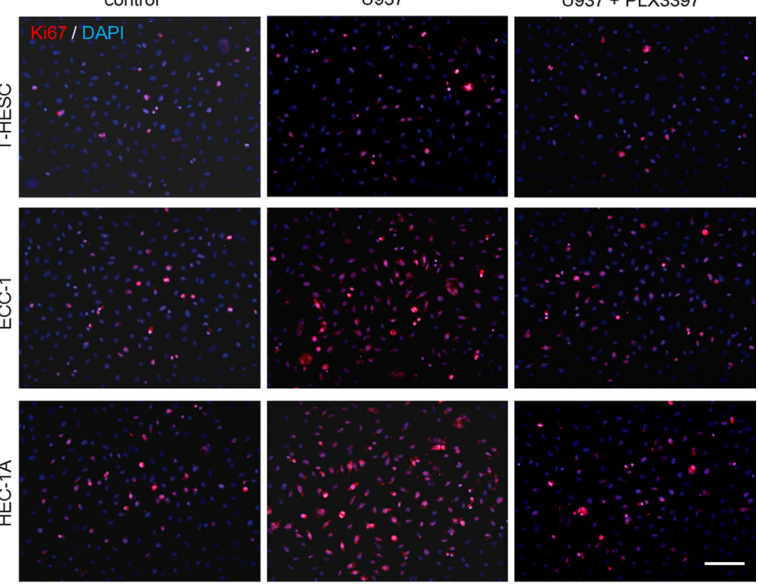

Figure 4. Blocking CSF-1R inhibits proliferation of endometrial cancer cells. (A) Immunofluorescence staining of M1 macrophage (iNOS and CD86) and M2 macrophage (Arg-1 and CD206) in U937 cell lines, co-cultured with ECC-1/HEC-1A cell lines and treated with 100 U/ml M-CSF. (B) Cell counting kit-8 assay found that U937 cells could promote ECC-1 and HEC-1A cell proliferation. Additionally, the CSF-1R inhibitor PLX3397 (10 $\mu$ M) inhibits proliferation of ECC-1 and HEC-1A cells in the co-culture system. (C) Immunofluorescence staining of Ki67 detecting EC cell proliferation. Data are presented as the mean \pm standard deviation from 5 independent experiments; ${ }^{*} \mathrm{P}<0.05,{ }^{* *} \mathrm{P}<0.01$ vs. Control. Scale bar: $50 \mu \mathrm{m}$. Arg, arginase; CD, cluster of differentiation; CSF, colony-stimulating factor; CSF-1R, colony-stimulating factor 1 receptor; EC, endometrial cancer; iNOS, inducible nitric oxide synthase.

culture system, the proliferation rate of endometrial cancer cells decreased, without affecting the proliferation of normal endometrial cells (Fig. 4B). Additionally, the proliferation of
EC cells in the co-culture system was investigated by Ki67 immunofluorescence staining. Consistent with the above conclusions, it was found that the proliferation of EC cells was 

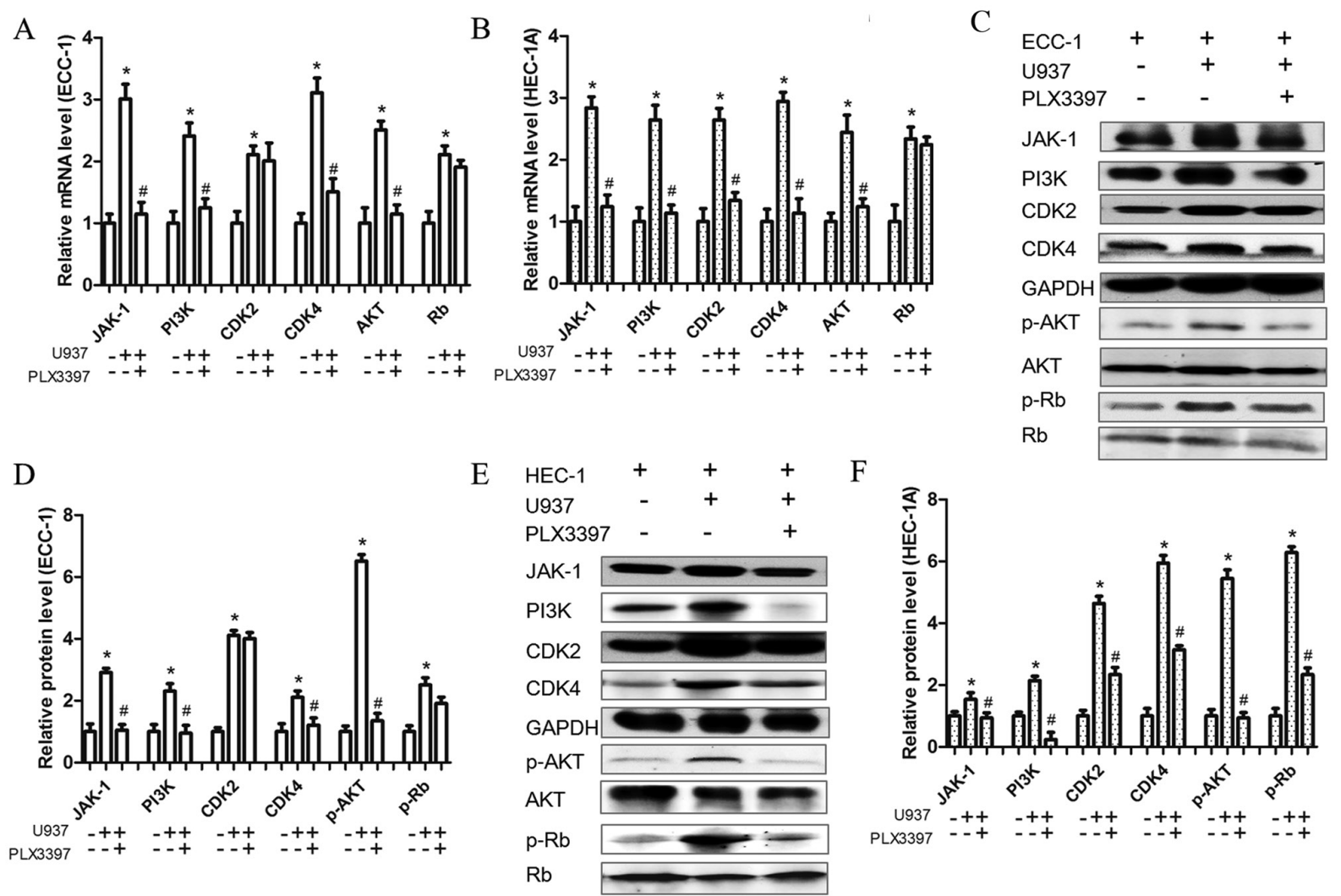

Figure 5. CSF-1R inhibitor influences proliferation-associated protein expression. (A and B) mRNA expression levels of JAK-1, PI3K, AKT, CDK2, CDK4 and Rb, in (A) ECC-1 and (B) HEC-1A cells and their inhibition by the CSF-1R inhibitor PLX3397 (10 $\mu \mathrm{M}$ ), as measured by reverse transcription-quantitative polymerase chain reaction. (C) Protein expression of JAK-1, PI3K, p-AKT, CDK2, CDK4 and p-Rb and (D) relative quantification of their expression levels in ECC-1 cells and their inhibition by the CSF-1R inhibitor PLX3397 $(10 \mu \mathrm{M})$, as measured by western blotting. (E) Protein expression of JAK-1, PI3K, p-AKT, CDK2, CDK4 and p-Rb and (F) relative quantification of their expression levels in HEC-1A cells and their inhibition by the CSF-1R inhibitor PLX3397 $(10 \mu \mathrm{M})$, as measured by western blotting. Data are presented as the mean \pm standard deviation from 5 independent experiments; ${ }^{*} \mathrm{P}<0.05 \mathrm{vs}$. Control; ${ }^{*} \mathrm{P}<0.05$ vs. U937 cells and ECC-1 or HEC-1A cells co-culture group. CDK4, cyclin-dependent kinase 4; CSF, colony-stimulating factor; CSF-1R, colony-stimulating factor 1 receptor; EC, endometrial cancer; JAK, Janus kinase; p, phosphorylated; PI3K, phosphoinositide 3-kinase; Rb, retinoblastoma-associated protein.

increased in the co-culture system, whereas it was inhibited by the CSF-1R inhibitor PLX3397 (Fig. 4C). Therefore, it was speculated that CSF-1 secreted by EC cells may promote migration of macrophages, transforming them to tumor-associated macrophages and that some growth factors secreted by tumor-associated macrophages promoted EC cells proliferation.

In order to further clarify the role of macrophages in promoting the proliferation of EC cells by CSF-1 and CSF-1R binding, the expression of proliferation-associated molecules was investigated at the mRNA and protein expression levels. It was found that U937 co-cultured with EC cells significantly increased the mRNA expression levels of JAK-1, PI3K, AKT, CDK2, CDK4 and Rb, however, their expression levels, apart from that of CDK2 (ECC-1 cells only) and Rb (ECC-1 and HEC-1A cells), were decreased when PLX3397 was pre-added in the co-culture system (Fig. 5A and B). Additionally, the protein expression levels of JAK-1, PI3K, p-AKT, CDK2, CDK4 and p-Rb were all increased in the co-culture system, and, apart from $\mathrm{p}-\mathrm{Rb}$ and CDK2 they all decreased when the CSF-1R was blocked (Fig. 5C-F). However, in the ECC-1 and U937 co-culture system, PLX3397 did not inhibit CDK2 expression at the mRNA or protein levels, whereas PLX3397 did not affect the expression of $\mathrm{Rb}$ at the mRNA level either in
ECC-1 and U937 co-culture system or in HEC-1A and U937 co-culture system. Consequently, it may be concluded that EC cells secreted CSF-1 to promote macrophage migration, which would then promote the proliferation of EC cells. On the other hand, when CSF-1R was blocked, the migration of macrophages and the proliferation of EC cells were both attenuated. However, this needs to be validated further.

\section{Discussion}

Although macrophages and other mononuclear phagocytes are regulated by a variety of growth factors, CSF-1 is still the most important regulator of TAM $(15,16)$. Previous studies found that CSF-1 and its receptors are mainly involved in the induction of monocyte development, and CSF-1 secreted by tumor (breast, ovarian and endometrial cancer) cells was found to promote tumor cell invasion (17). The present study found that CSF-1 secreted by EC cells binds to CSF-1R located on the surface of macrophages, inducing macrophage infiltration, promoting the proliferation of EC cells. Macrophages are divided into M1 type (classic activated macrophages) and M2 type (alternatively activated macrophages) (18). M1 macrophages are involved in the inflammatory response, clear the body from pathogens, participate in antitumor immunity, whereas M2 macrophages have the function of 
anti-inflammatory, repair damage and they promote tumor formation (19). Macrophages infiltrate into malignant tumor tissues in high numbers and it has been demonstrated that macrophages have pro-tumor functions and are closely related to tumor progression. Consistent with these functions, studies using human tumor samples have demonstrated that a higher density of macrophages, especially macrophages with the M2 phenotype, is closely associated with worse clinical prognosis in many kinds of malignant tumors (20-22). In the early stages of tumorigenesis, TAM takes M1 macrophages as the main body and serves the role of killing tumor cells, whereas in the late stages of tumor development, TAM is mainly consisted by M2 macrophages, which could promote tumor growth, invasion and metastasis (23). CSF-1 can promote macrophage survival and differentiation, and macrophages can be converted to TAM in its presence (12). Therefore, in the present study, when exogenous CSF-1 was added to the macrophage culture medium, or macrophages and EC cells were co-cultured, macrophages were transformed into TAM favoring the growth of EC cells, therefore, macrophages seem to have induced the proliferation of EC cells in the co-culture system, but this needs to be verified in future studies.

In the present study, EC cells promoted the migration of macrophages by secreting CSF-1, and macrophages did not promote cell proliferation after blocking the CSF-1R. The above results demonstrated that under the presence of CSF-1, macrophages undergo phenotypic conversion, as EC cells convert macrophages to TAM which prompting EC cells to proliferate more. Therefore, targeting CSF-1 to reduce the number of TAMs can be used as a cancer treatment. Zeisberger et al (24) used liposome-coated clodronate to remove macrophages from the whole body of tumor-bearing mice, showing that tumor growth was effectively controlled. In a study of murine breast cancer induced by spontaneous polyoma virus middle $\mathrm{T}$ oncoprotein, Lin et al (25) demonstrated that transgenic mice deficient in CSF-1 could significantly reduce the number of TAMs, reduce tumor angiogenesis and delay tumor growth progression reducing lung metastases. Previous studies have pointed out that the removal of CSF-1 and other factors from the ascites of ovarian cancer would affect the macrophage-induced TAM $(26,27)$. Other studies have used transgenic technology to overexpress CSF-1 in mammary glands, showing that the branches of breast ducts increased and precancerous lesions appeared, suggesting that CSF-1 promotes tumorigenesis (28). Other studies have indicated that CSF-1/CSF-1R-induced activation of nuclear factor- $\mathrm{\kappa B}$ in TAM is required for tumor progression in inflammation-induced murine tumor models $(29,30)$. Staining of CSF-1 and its receptor showed strong positive immunostaining in metastatic ovarian carcinoma, while benign ovarian tissue showed a little expression of CSF-1/CSF-1R (31). Therefore, high expression of CSF-1 and its receptor regulates the proliferation of tumor cells. At present, there are many strategies and methods for tumor therapy by targeting TAM; however, the role of TAM in EC and by which way EC cells recruit macrophage is still unclear. The present study demonstrated that EC cells can recruit macrophages by secreting CSF-1, and macrophages stimulated by CSF-1 can promote the proliferation of EC cells. The development of drugs targeting TAM, especially humanized antibodies or inhibitors targeting CSF-1 or its receptor, promise benefits in cancers such as EC.

\section{Acknowledgements}

The authors would like to thank Professor Ren Mulan, the Dean of Gynecology, Southeast University (Jiangsu, China), for his individual support and help to this study.

\section{Funding}

No funding was received.

\section{Availability of data and materials}

The datasets used and/or analyzed during the current study are available from the corresponding author on reasonable request.

\section{Authors' contributions}

FH and YT performed the experiments and analyzed the data. YG analyzed the data. CL and XL collected EC tissues of patients and $\mathrm{FH}$ prepared the manuscript.

\section{Ethics approval and consent to participate}

The present study was approved by the Ethics Committee of Nanjing Medical University and written informed consent was obtained from each patient prior to surgery and enrolment in the study.

\section{Patient consent for publication}

Patients consented to the publication of their clinical data.

\section{Competing interests}

The authors declare that they have no competing interests.

\section{References}

1. van Niekerk CC, van Dijck JAAM and Verbeek ALM: The impact of histological subtype in developing both ovarian and endometrial cancer: A longstanding nationwide incidence study. Eur J Obstet Gynecol Reprod Biol 221: 17-22, 2018.

2. Morice P, Leary A, Creutzberg C, Abu-Rustum N and Darai E: Endometrial cancer. Lancet 387: 1094-1108, 2016.

3. Lewis CE and Pollard JW: Distinct role of macrophages in different tumor microenvironments. Cancer Res 66: 605-612, 2006.

4. Yuan A, Chen JJ and Yang PC: Pathophysiology of tumor-associated macrophages. Adv Clin Chem 45: 199-223, 2008.

5. Hu HL, Bai HS and Pan HX: Correlation between TAMs and proliferation and invasion of type I endometrial carcinoma. Asian Pac J Trop Med 8: 643-650, 2015.

6. Kelly MG, Francisco AM, Cimic A, Wofford A, Fitzgerald NC, Yu J and Taylor RN: Type 2 endometrial cancer is associated with a high density of tumor-associated macrophages in the stromal compartment. Reprod Sci 22: 948-953, 2015.

7. Tong H, Ke JQ, Jiang FZ, Wang XJ, Wang FY, Li YR, Lu W and Wan XP: Tumor-associated macrophage-derived CXCL8 could induce ER $\alpha$ suppression via HOXB13 in endometrial cancer. Cancer Lett 376: 127-136, 2016.

8. Soeda S, Nakamura N, Ozeki T, Nishiyama H, Hojo H, Yamada H, Abe M and Sato A: Tumor-associated macrophages correlate with vascular space invasion and myometrial invasion in endometrial carcinoma. Gynecol Oncol 109: 122-128, 2008. 
9. Espinosa I, Catasus L, D' Angelo E, Mozos A, Pedrola N, Bértolo C, Ferrer I, Zannoni GF, West RB, van de Rijn M, et al: Stromal signatures in endometrioid endometrial carcinomas. Mod Pathol 27: 631-639, 2014

10. Mantovani A and Sica A: Macrophages, innate immunity and cancer: Balance, tolerance, and diversity. Curr Opin Immunol 22 . 231-237, 2010.

11. O'Brien J, Lyons T, Monks J, Lucia MS, Wilson RS, Hines L, Man YG, Borges V and Schedin P: Alternatively activated macrophages and collagen remodeling characterize the postpartum involuting mammary gland across species. Am J Pathol 176: $1241-1255,2010$

12. Pyonteck SM, Akkari L, Schuhmacher AJ, Bowman RL, Sevenich L, Quail DF, Olson OC, Quick ML, Huse JT, Teijeiro V, et al: CSF-1R inhibition alters macrophage polarization and blocks glioma progression. Nat Med 19: 1264-1272, 2013.

13. Livak KJ and Schmittgen TD: Analysis of relative gene expression data using real-time quantitative PCR and the 2(-Delta Delta C(T)) method. Methods 25: 402-408, 2001

14. Dou F, Huang L, Yu P, Zhu H, Wang X, Zou J, Lu P and Xu XM: Temporospatial expression and cellular localization of oligodendrocyte myelin glycoprotein (OMgp) after traumatic spinal cord injury in adult rats. J Neurotrauma 26: 2299-2311, 2009.

15. Murray PJ and Wynn TA: Protective and pathogenic functions of macrophage subsets. Nat Rev Immunol 11: 723-737, 2011.

16. Hamilton JA: Colony-stimulating factors in inflammation and autoimmunity. Nat Rev Immunol 8: 533-544, 2008.

17. Kacinski BM: CSF-1 and its receptor in ovarian, endometrial and breast cancer. Ann Med 27: 79-85, 1995.

18. Dun EC, Hanley K, Wieser F, Bohman S, Yu J and Taylor RN: Infiltration of tumor-associated macrophages is increased in the epithelial and stromal compartments of endometrial carcinomas. Int J Gynecol Pathol 32: 576-584, 2013.

19. Sica A and Mantovani A: Macrophage plasticity and polarization: In vivo veritas. J Clin Invest 122: 787-795, 2012.

20. Weigert A, Sekar D and Brune B: Tumor-associated macrophages as targets for tumor immunotherapy. Immunotherapy 1: 83-95, 2009.

21. Jinushi M and Komohara Y: Tumor-associated macrophages as an emerging target against tumors: Creating a new path from bench to bedside. Biochim Biophys Acta 1855: 123-130, 2015.
22. Komohara Y, Fujiwara Y, Ohnishi K and Takeya M: Tumor-associated macrophages: Potential therapeutic targets for anti-cancer therapy. Adv Drug Deliv Rev 99: 180-185, 2016.

23. Ruffell B, Affara NI and Coussens LM: Differential macrophage programming in the tumor microenvironment. Trends Immunol 33: 119-126, 2012.

24. Zeisberger SM, Odermatt B, Marty C, Zehnder-Fjällman AH, Ballmer-Hofer K and Schwendener RA: Clodronate-liposome-mediated depletion of tumour-associated macrophages: A new and highly effective antiangiogenic therapy approach. Br J Cancer 95: 272-281, 2006.

25. Lin EY, Nguyen AV, Russell RG and Pollard JW: Colony-stimulating factor 1 promotes progression of mammary tumors to malignancy. J Exp Med 193: 727-740, 2001.

26. Duluc D, Delneste Y, Tan F, Moles MP, Grimaud L, Lenoir J, Preisser L, Anegon I, Catala L, Ifrah N, et al: Tumor-associated leukemia inhibitory factor and IL-6 skew monocyte differentiation into tumor-associated macrophage-like cells. Blood 110: 4319-4330, 2007.

27. Jeannin P, Duluc D and Delneste Y: IL-6 and leukemia-inhibitory factor are involved in the generation of tumor-associated macrophage: Regulation by IFN- $\gamma$. Immunotherapy 3 (4 Suppl): S23-S26, 2011.

28. Kirma N, Hammes LS, Liu YG, Nair HB, Valente PT, Kumar S, Flowers LC and Tekmal RR: Elevated expression of the oncogene c-fms and its ligand, the macrophage colony-stimulating factor-1, in cervical cancer and the role of transforming growth factor-betal in inducing c-fms expression. Cancer Res 67: 1918-1926, 2007.

29. Mancino A and Lawrence T: Nuclear factor-kappaB and tumor-associated macrophages. Clin Cancer Res 16: 784-789, 2010.

30. Biswas SK and Lewis CE: NF- $\kappa \mathrm{B}$ as a central regulator of macrophage function in tumors. J Leukoc Biol 88: 877-884, 2010.

31. Chambers SK: Role of CSF-1 in progression of epithelial ovarian cancer. Future Oncol 5: 1429-1440, 2009.

(i) () $(9)$ This work is licensed under a Creative Commons

Attribution-NonCommercial-NoDerivatives 4.0 International (CC BY-NC-ND 4.0) License. 